\title{
Research on Rapid Detection Method of Airborne Transmission lines Based on Salient Region Detection
}

\author{
Hongdi $\mathrm{Ke}^{1, \mathrm{a} *}$ and Ming Lu ${ }^{1, \mathrm{~b}}$ \\ ${ }^{1}$ Department of Information Engineering, Jilin Business and Technology College, Changchun, \\ China \\ a13661033@qq.com,'p75579008@qq.com
}

Keywords: Salient Region Detection; LC Algorithm; HC Algorithm; FT model; Aerial Transmission Lines

\begin{abstract}
From the perspective of machine vision, this paper studied the salient area detection method of aerial transmission line image, used LC algorithm, HC algorithm, FT model to detect the salient area of air transmission line. We used MATLAB to simulate them, and results showed that FT model is better than the others.
\end{abstract}

\section{Introduction}

Transmission line is an important part of the national power grid, it is an important work to ensure the safety and normal transmission of transmission lines by periodical inspection ${ }^{[1]}$. The traditional artificial ground patrol line operation has high labor intensity and dangerous coefficient, especially in plateau and mountainous area.

With the wide application of UAV, it is a trend for future transmission line patrol to replace the unmanned aerial vehicle (UAV) with the manual application. For the processing of patrol image, the extraction and recognition of target image is a key technology ${ }^{[2]}$. Because of the complexity and diversity of the background, the difference between the target image and the background image is very small, so the extraction of the target image and the removal of the background are the bottleneck of the patrol image processing ${ }^{[3]}$.

From the view of machine vision, the feature point distribution in the feature detection phase of the local feature extraction algorithm is used to detect the salient region of the image quickly, then the cluster analysis theory is applied to the anomaly detection of the aerial transmission line patrol image, and the characteristic value of the salient region is extracted by MATLAB.It is possible to quickly isolate the key components of the transmission line from the complex background (insulators, spacer rod), it is important to establish a more efficient image segmentation algorithm to identify the critical component anomalies and to deal with the faults in time, and to improve the identification and fault detection ability of transmission lines and key components in helicopter patrol images.

\section{The Salient Region Detection Method}

There are a lot of salient region detection methods, such as: IT model, MZ model, GB model and so on, this paper uses LC algorithm 、 HC algorithm、FT model to study the salient area detection of aerial transmission line images.

LC Algorithm. The LC algorithm uses the color statistic information of the image to extract the salient map of the space. Based on the histogram, the algorithm mainly considers the color global contrast, the computational complexity is linearly correlated with the pixel number of the image[4,5], and the calculation of the salient value shows as follow.

The salient value of a pixel Ik in image I is calculated as follows:

$$
\operatorname{SalS}\left(\mathrm{I}_{\mathrm{k}}\right)=\sum_{\forall \mathrm{I}_{\mathrm{i}} \in \mathrm{I}}\left\|\mathrm{I}_{\mathrm{k}}-\mathrm{I}_{\mathrm{i}}\right\|
$$

The range of the Ii value is $[0,255]$, which is the gray value.

Eq. 1 equals: 


$$
\operatorname{SalS}\left(\mathrm{I}_{\mathrm{k}}\right)=\left\|\mathrm{I}_{\mathrm{k}}-\mathrm{I}_{1}\right\|+\left\|\mathrm{I}_{\mathrm{k}}-\mathrm{I}_{2}\right\|+\cdots+\left\|\mathrm{I}_{\mathrm{k}}-\mathrm{I}_{\mathrm{N}}\right\|
$$

$\mathrm{N}$ represents the number of pixels in the image, Given an image, the color value of $\mathrm{I}_{\mathrm{k}}$ per pixel is known.Assuming $\mathrm{I}_{\mathrm{k}}=\mathrm{a}_{\mathrm{m}}$, Eq. 2 can be further refactored:

$$
\begin{aligned}
& \operatorname{SalS}\left(\mathrm{I}_{\mathrm{k}}\right)=\left\|\mathrm{a}_{\mathrm{m}}-\mathrm{a}_{0}\right\|+\cdots+\left\|\mathrm{a}_{\mathrm{m}}-\mathrm{a}_{1}\right\|+\cdots+ \\
& \operatorname{SalS}\left(\mathrm{a}_{\mathrm{m}}\right)=\sum_{\mathrm{n}=0}^{255} \mathrm{f}_{\mathrm{n}}\left\|\mathrm{a}_{\mathrm{m}}-\mathrm{a}_{\mathrm{n}}\right\|
\end{aligned}
$$

The $f_{n}$ represents the frequency of the nth pixel in the image which expressed in the form of a histogram.

HC Algorithm. In essence, HC algorithm is no different from the LC algorithm, but HC algorithm considers color information, rather than using pixel-only gray scale information like LC algorithm, because the color image has the most $256^{*} 256^{*} 256$ color, so the direct histogram based scheme is not feasible.

But in fact a color image does not use so many colors, therefore, the author proposed to reduce the number of colors of the scheme, the RGB components are mapped into 12 equal parts, the insinuate after the graph is only $12 * 12 * 12$ color, so you can construct a smaller histogram to accelerate, However, because of the transition quantization will bring some flaws in the results. So the author used a smooth process. Finally, unlike the LC, the author's processing in the lab space, and because the lab space and RGB is not exactly the corresponding, the quantization process is still in the RGB space to complete ${ }^{[4-6]}$.

Analysis of the salient regions of color image pixels by the $\mathrm{HC}$ algorithm:

$$
\mathrm{S}(\mathrm{p})=\mathrm{S}\left(\mathrm{C}_{\mathrm{i}}\right)=\sum_{\mathrm{i}=1}^{\mathrm{n}} \mathrm{f}_{\mathrm{i}} \mathrm{d}\left(\mathrm{c}_{1}, \mathrm{c}_{\mathrm{i}}\right)
$$

The color value of $\mathrm{P}$ is $\mathrm{c}_{1}$, fi the frequency of $\mathrm{C}_{\mathrm{I}}$ in above image, and by looking at the histogram, $\mathrm{n}$ is the total amount of other colors, $\mathrm{D}(\cdot, \cdot)$ is the distance between the two colors in CIELAB space.

The Eg.5 calculation cost is $\mathrm{O}(\mathrm{N})+\mathrm{O}\left(\mathrm{n}^{2}\right)$, which is very large for natural images, and the $\mathrm{HC}$ model uses color histogram to accelerate. First in the RGB space to quantify the color, three color channels in each channel to retain only 12 different values, so that the color of the image will be greatly reduced.Then discard some colors that appear less likely in most natural images, the final HC model uses only 85 colors, and the few pixel colors in the natural image that are not accurately expressed are replaced with the approximate colors in the 85 colors above.

FT Model. Frequency domain tuning Model FT focuses on the analysis of the effect of frequency on the Salient Region Detection, it attempts to retain more image frequency information, and then extract a better definition of the boundaries of High-quality objects.

The $\omega_{\mathrm{LC}}$ and $\omega_{\mathrm{HC}}$ are the low-frequency and high-frequency thresholds of the images respectively. To highlight a larger object, we need to emphasize the low-frequency information of the original graph, and the threshold $\omega_{1}$ should be as low as possible, which also helps to make the significant degree of the various parts of the object more coherent $t^{[7,8]}$.

To obtain a high degree of uniformity of the boundary, should retain the original image of high-frequency information, threshold $\Omega \mathrm{HC}$ higher. But to shield off the highest band, to remove noise, coding traces, textures and so on. The FT model is interested in the middle part of low-frequency threshold $\omega_{\mathrm{LC}}$ and high-frequency threshold $\omega_{\mathrm{HC}}$.

The FT model uses the Gaussian differential band pass filter (dog), which is good at perceiving the changes of gray level and is widely used in computer vision tasks such as edge detection ${ }^{[9,10]}$.

$$
\operatorname{DoG}(\mathrm{i}, \mathrm{j})=\frac{1}{2 \pi}\left[\frac{1}{\sigma_{1}^{2}} \exp \left(-\frac{\left(\mathrm{i}^{2}+\mathrm{j}^{2}\right)}{2 \delta_{1}^{2}}\right)-\frac{1}{\sigma_{2}^{2}} \exp \left(-\frac{\left(\mathrm{i}^{2}+\mathrm{j}^{2}\right)}{2 \delta_{2}^{2}}\right)\right]=\mathrm{G}\left(\mathrm{i}, \mathrm{j}, \sigma_{1}\right)-\mathrm{G}\left(\mathrm{i}, \mathrm{j}, \sigma_{2}\right)
$$

$\sigma_{1} 、 \sigma_{2}$ is the Gaussian standard deviation, DoG bandwidth controls by $\sigma_{1}, \sigma_{2}$, when

$\sigma_{1}: \sigma_{2}=1: 1.6$ the best detection effect. The $\mathrm{P}=\sigma_{1} / \sigma_{2}, \mathrm{FT}$ model combines many DoG filters to increase the transmission bandwidth. 


$$
\mathrm{FT}_{\mathrm{N}}=\sum_{\mathrm{n}=0}^{\mathrm{N}-1} \mathrm{G}\left(\mathrm{i}, \mathrm{j}, \rho^{\mathrm{n}+1} \sigma\right)-\mathrm{G}\left(\mathrm{i}, \mathrm{j}, \rho^{\mathrm{n}} \sigma\right)=\mathrm{G}\left(\mathrm{i}, \mathrm{j}, \delta_{1}\right)-\mathrm{G}\left(\mathrm{i}, \mathrm{j}, \delta_{2}\right)
$$

For $\mathrm{FT}_{\mathrm{N}}, \delta_{1}=\rho^{\mathrm{n}} \delta, \delta_{2}=\delta$. The FT model allows $\delta_{1}$ infinity and $\mathrm{G}\left(\mathrm{i}, \mathrm{j}, \rho^{\mathrm{n}} \delta\right)$ to compute the mean value of the image $\mathrm{I}_{\mu}, \delta_{2}$ using a Gaussian core $1 / 16[1,4,6,4,1$ with a window size of $5 \times 5$, which can be used to represent a formula as Eq.8.

$$
\mathrm{S}(\mathrm{i}, \mathrm{j})=\left\|I_{\mu}-I_{w h c}(i, j)\right\|
$$

The $I_{w h c}$ is a surprise Gaussian smoothing image of the original image, with some detail textures, noises and coding impairments removed.The FT model uses CIELAB space to set the eigenvector for each pixel and $\|\cdot\|$ the Euclidean distance representing the eigenvector.

\section{MATLAB Simulation}

LC Algorithm Realize. We used MATLAB to Simulate LC algorithm as follow.

First:calculate the histogram of the image, you can get each gray level of the corresponding number of pixels. Gray level $[0,255] .1 * 256$ Matrix record.

Second:calculate each characteristic value $\mathrm{P}(0 \sim 255)$ significant value, $1 * 256$ matrix record.

Third: Assign a significant value to each pixel.(I,J) is what gray level gives it the corresponding gray level of the significant value.Get a significant figure.

Last: to the salient map of normalization.Get the final significant figure.

LC algorithm is simple, efficient, computationally time-consuming, can be used in many computer vision tasks, such as rapid scene analysis, but in some cases, too much emphasis on some rare colors, affecting the quality of the significance of the analysis.

HC Algorithm Realize. We used MATLAB to Simulate HC algorithm, the above quantitative acceleration method can greatly improve the time performance of the model, but it will reduce the accuracy of the significant detection, introduce some artificial traces, use the N/4 technique to solve the problem, and use a variety of colors (color) similar color to optimize the significance of the color measurement.

$$
\begin{aligned}
& \dot{\mathrm{S}}(\mathrm{c})=\frac{1}{\left(\frac{\mathrm{n}}{4}-1\right) \mathrm{T}} \sum_{\mathrm{i}=1}^{\frac{\mathrm{n}}{4}}\left(\mathrm{~T}-\mathrm{d}\left(\mathrm{c}, \mathrm{c}_{\mathrm{i}}\right)\right) \mathrm{S}\left(\mathrm{c}_{\mathrm{i}}\right) \\
& \mathrm{T}=\sum_{\mathrm{i}=1}^{\mathrm{n} / 4} \mathrm{~d}\left(\mathrm{c}, \mathrm{c}_{\mathrm{i}}\right)
\end{aligned}
$$

The HC algorithm is quickly, the detection effection is accurate and complete, and it can produce a significant graph with full resolution, but it can't deal with the image with more complicated texture.

FT Model Realize. We used MATLAB to Simulate FT model. The FT model is simple to implement, the computation is small, the edge of the object is more fitting, the quality is high, and it is applied to many computer vision tasks such as significant object segmentation.

\section{Summary}

The LC algorithm, HC algorithm and FT model are applied to the salient region detection of aerial transmission line image, through MATLAB simulation, table 1 lists LC algorithm, HC algorithm, FT model three methods to compare the salient region detection results of aerial transmission line images. 
Table 1 Comparison of salient region detection results of three algorithms

\begin{tabular}{|l|l|l|l|}
\hline & LC algorithm & HC algorithm & FT model \\
\hline Simple to achieve & yes & yes & yes \\
\hline Calculation speed & fast & fast & fast \\
\hline Detection effect & good & better & best \\
\hline $\begin{array}{l}\text { Processing complex } \\
\text { images of textures }\end{array}$ & no & no & no \\
\hline
\end{tabular}

Table 1 shows that, in terms of the degree of implementation of the salient area detection, LC algorithm, HC algorithm and FT model are all Simple to achieve. in terms of the degree of Calculation speed, LC algorithm, HC algorithm and FT model are all fast. in terms of the degree of detection effect, FT model is better than the others.but in terms of the degree of Processing complex images of textures, LC algorithm, HC algorithm and FT model are all bad.

We studied salient area detection method of aerial transmission line image from the perspective of first ,we introduced the principle of LC algorithm, HC algorithm, FT model ,then we used LC algorithm, HC algorithm, FT model to detect the salient area of air transmission line. MATLAB simulation results showed that FT model is better than the others.

\section{Acknowledgements}

The project was supported by the Jilin Provincial Education Department's Planning Project (JJKH20180517KJ) and the PhD Fund (KB2018[007) of Jilin Business College.

\section{References}

[1] K.Zhang, H.F.Li and W.Wang:High Voltage Technology, Vol.6.(2013), No.32: p45.

[2] P.Wang, G.Li and K.Zhu: Sichuan Electric Power Technology, Vol.2.(2012), No.10: p4.

[3] D.M.Yu, J.Shen and J.Wang: Power Grid Technology, Vol.6.(2009), No.33: p107.

[4] Information on https://blog.csdn.net/wan gyaninglm/article/details/44020489.

[5] Information on https://asdfv1929.github.io/2018/05/11/saliency-LC/c.

[6] Information https://wenku.baidu.com/view/cfac804110a6f524ccbf85a5.html?rec_flag=default\&sxts=15307 15307891

[7] X.D.Hou,J.Harel and C.Koch: IEEE Transaction on Pattern Analysis and Machine Intelligence. Vol.1.(2012), No.34: p194.

[8] N.Ouerhani,R.von Wartburg,H.Hugli and R.Muri.Empirical:Electronic Letters on ComputerVision and Image Analysis. Vol.1.(2004), No.3: p13.

[9] L. J. Duan, C. P. Wu, J. M, L. Y. Qing and Y. Fu:.In Proceedings of the IEEE Conference on Computer Visionand Pattern Recognition( New York, America, 2011). Vol. p473.

[10] B. Han, H. Zhu and Y. Ding:In Proceedings of the ACM International Conference on Multimedia( New York, America, 2011) Vol. p1117. 